\title{
Flow field velocity measurement of liquid interaction with rigid and flexible wall
}

\author{
Darina Jasikova ${ }^{l},{ }^{*}$, Michal Kotek $^{l}$, Frantisek Pochyly $^{2}$, and Vaclav Kopecky ${ }^{1}$ \\ ${ }^{1}$ Department of physical measurements, Institute for Nanomaterials, Advanced Technology and Innovation, Studentska 1402/2 \\ 46001 Liberec 1, Czech Republic \\ ${ }^{2}$ Victor Kaplan Dept. of Fluid Engineering, Faculty of Mechanical Engineering, Brno university of technology, Technicka 2896/2, 616 \\ 69 Brno, Czech Republic
}

\begin{abstract}
The motivation of this research was to determine the flow interactions on the pulsation and to express the influence on the flow character in the rigid and flexible tube. The character of Newtonian liquid was measured with the Particle Image Velocimetry method (PIV). Here, we used glass tube and Tygon tube for our comparison. We build the circuit equipped with membrane pump for generating pulsatile flow. The results were analysed over the pulse period sampled in 10 time steps. The fluid flow varied from $\operatorname{Re} 560$ to Re 8800. The velocity profiles uncovered backward revers flows closed to the wall. These structures are prevailing close to flexible wall. The effect of interaction between pulsatile liquid flow and flexible wall was experimentally proved.
\end{abstract}

\section{Introduction}

Traditionally flows are studied and analysed in rigid tubes as continuous flow. There is increasing need for study fluid flow characteristics in flexible tubes. The typical applications are bio-technological processes, i.e. separation and purification as well as development of artificial organ replacements. Dynamics of fluid flow past flexible solid surfaces is qualitatively different task to solve.

Next step in studying the flow in flexible walls is the use of a pulsatile pump. Here we aim for arterial replacements or external blood circulation. McDonald studied the relation of pressure to flow as haemodynamic problem. [1, 2] An exact solution of the equations of viscous fluid motion in a circular tube under a pressure gradient as a periodic function of the time was brought by Womersley. These experiments run on real dog's and rabbit's arteries using basic measurement sensors for flow anemometry and pressure. It has been shown that the characteristic pulsatile flow across the flexible tube is dependent on the waveform. A reference number known as Womersley number has been determined. Womersley number $\alpha$ is set.

$$
\propto^{2}=\frac{\text { transient internal force }}{\text { viscous force }}=\frac{\rho \omega U}{\mu U L^{-2}}=\frac{\omega L^{2}}{v}
$$

the Womersley number can be expressed as:

$$
\alpha=L \sqrt{\frac{2 \pi f \cdot \rho}{\mu}}
$$

where $\omega$ is angular frequency, $f$ is the ordinary frequency, $L$ is an appropriate length scale (radius of a pipe), $v$ is fluid kinematic viscosity, $\mu$ is fluid dynamic viscosity. It expresses the ratio of the transient or oscillatory inertia force to the shear force. When $\alpha<1$, there is developed a typical parabolic velocity profile during each cycle, and there will be given to a good approximation by Poiseuille's law, using the instantaneous pressure gradient. A flat velocity profile is developed, when $\alpha>10$. [1-4] This condition is corresponding with turbulent zone that can be expected in a reaction on pressure wave. In our experiment, according to eq.2, the radius of tube was $1 \mathrm{~cm}$, working with water of density $0.99753 \mathrm{~g} / \mathrm{cm}^{3}\left(23^{\circ} \mathrm{C}\right)$, and the angular frequency $2 \pi f$ for $f=1 \mathrm{~Hz}$, the Womersley number is 27,98 . This means that the ratio number is close to the human ascending aorta of radius $1,25 \mathrm{~cm}$, and heart beat frequency of $3 \mathrm{~Hz}$ that is 27,13 . This expression gives us the opportunity to carry the experiment towards the situation in real physiological biological system.

The determination takes into account time averaging, but if we have a pulsatile flow, the velocity profile is different with respect to course of pressure. Here it is important to note that Womersley number expresses a relationship pronouncing two systems of different diameters (radius), fluid properties, and pulsatile flow that could be compare. This comparison is between the tube system (glass, and Tygon tubes) with pulsation of $1 \mathrm{~Hz}$, and human ascending aorta on real pulsation rate in our experiment, taking in account fluid properties, i.e. blood vs. water.

The cross-section velocity profile depends on the elasticity of the wall as well on the fluid properties. In this task, the elastic - flexible wall reacts to the change of pressure, which produces a feedback response that is reflected in the character of the nearby flow profile. [5]

Corresponding author: darina.jasikova@tul.cz 
However, this cannot be determined by conventional measurement techniques.

The motivation of this research was to determine the flow interactions on the pulsation and to express the influence of the elastic - flexible wall on the flow character. A suitable method for monitoring and evaluating changes in flow is the Particle Image Velocimetry method (PIV).

\section{Measurement Setup}

Water circuit from PVC-U pipes was designed and manufactured for testing the flow fields in different types of tubes. Here we used pure water for the purpose of this initial measurement as a medium. The pipes from PVC$\mathrm{U}$ made by Georg Fischer are solid enough not to influent or dump the pulsations in the circuit. The testing section was removable and suitable for fitting with glass and Tygon tubes. Both tubes have the diameter of 20 $\mathrm{mm}$. The glass tube is a representative of full-solid rigid tube. The Tygon tube is the representative of flexible wall tube. These tubes are made from flexible polymer, mostly used in medicine, food industry, chemical processing etc. The wall thickness of Tygon tube was $2 \mathrm{~mm}$.

Water in the circuit was actuated by the membrane pump (MP) Bran+Luebbe ProCam Smart DS500. The pump was controlled with the frequency driver (FD) Emerson Control Techniques Unidriver M200 to set optimal pulsation rate of $1 \mathrm{~Hz}$. Upper water tank was connected to the circuit to set up the initial pressure in the circuit. After that the tank was disconnected, so the pulsations run only in the circuit and were not dumped in the tank.

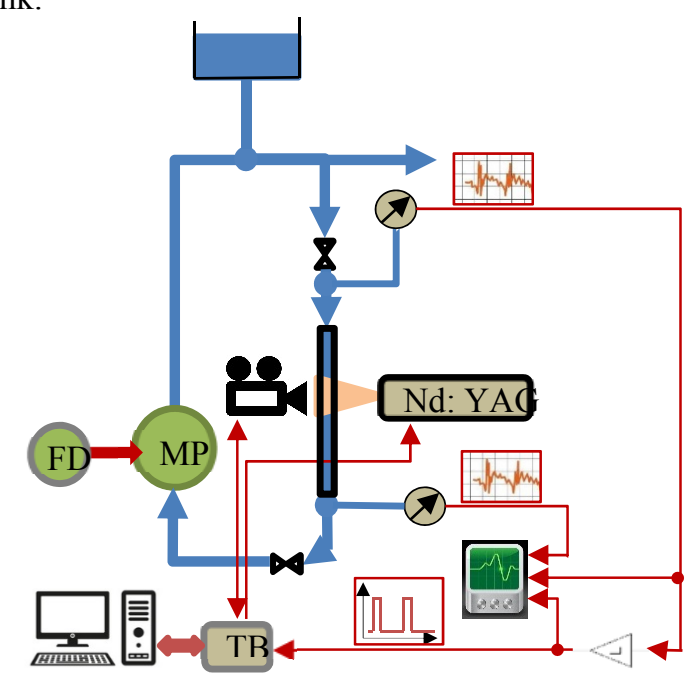

Fig. 1. Principle scheme of the water circuit with actuators, sensors, and PIV system. FD - frequency driver, MP membrane pump, TB - PIV Timer box, Nd: YAG - pulse laser

The circuit was equipped with two pressure sensors just below (Pressure Sensor 1) and above (Pressure Sensor 2) the observed tube. Sensor's analogue outputs were filtered, connected to the digital oscilloscope and saved for following analysis. Output from the above pressure sensor was also used as a trigger for PIV measurement. When the pressure value reached the proper value, the flip-flop circuit generated the TTL signal. That signal connected to the Timer Box (TB) triggered the PIV system with the required period shift (delay of the signal). Using different period shifts we provided ten repeatable measurements inside the pulse period. The trigger TTL signal was also connected and saved in the oscilloscope to verify correct timing of the measurement. With this setup we can reconstruct the behaviour of the flow and the pressure conditions in the water circuit simultaneously.

The PIV data were recorded using system from Dantec Dynamics and its HiSense Neo camera. The flow was illuminated by the pulse Nd:YAG laser NewWave Gemini (532 nm wavelength). One synchronised (period shifted) image was recorded in each period, so the frequency of the PIV system was also $1 \mathrm{~Hz}$. We collected 250 images for each time stamp in the period; the period was sampled with $100 \mathrm{~ms}(10$ samples in the pulse period). There were used Polyamide fluorescent particles (coloured with Rhodamine B with emitting wavelength of $570 \mathrm{~nm}$, size $5 \mu \mathrm{m}$ ) as seeding particles. The fluorescent particles enable measurement close to wall as the laser light scattered from the wall is eliminated. The principle scheme of the measurement circuit and PIV system set-up is seen on Figure 1.

The PIV measurements were made in the measuring plane lighted by a laser cut in the middle of the tube. The dataset of images were processed using dewarping method. Differences of refractive indices between several materials (water, glass/Tygon, air) cause image distortion. This distortion causes erroneous evaluation of the velocity fields, especially near the wall, that is, the area of our interest. The image calibration was performed before the measurement using a chessboard calibration target placed inside the tube and filled with water. The datasets of measurements were analysed using Cross-Correlation method (using Interrogation Area of 64 px x 64 px with 50\% overlap in both directions) and valuated with Moving Average Validation (Averaging matrix $3 \times 3$, without substituting vectors, and validation on boundaries).

\section{Results and Discussion}

Pulse flow was generated by a membrane pump. The nature of the pulsatile flow was measured with pressure sensors. We got information on the pressure development inside the circuit. Each phase of the pulse was evenly divided into 10 equal time slots. The individual slots were synchronized to the initial pressure change. The minimal pressure change was set at 0.14 bar. The charastic pressure recording is seen in Fig. 2.

The evaluation of the pressure measurement has already revealed the backward effect of the flexible wall. Especially when compared to glass rigid wall. The pulsation of the liquid is partially absorbed by the elastic wall. The energy is again released into the flowing fluid in the second phase of the pressure wave. These interactions will be shown as the oscillations of the 
pressure curve (Pressure Sensor 1) in the chart of the pressure time course as it is seen in Fig. 3.

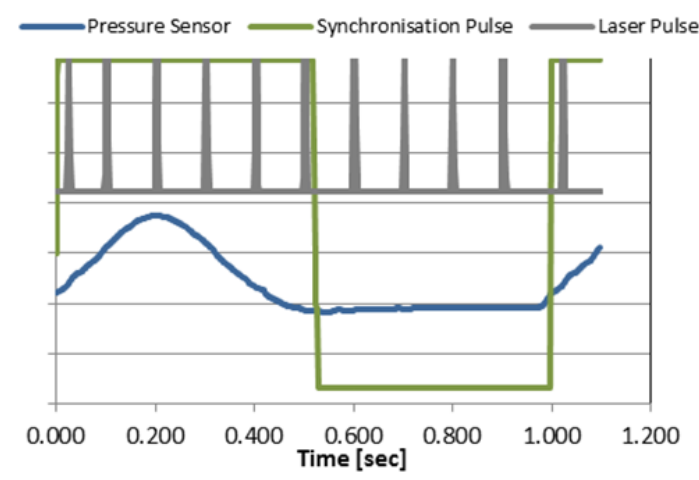

Fig. 2. Scheme of synchronization. The green line represents the synchronization pulse; the grey line represents 10 time slots for PIV measurement using laser pulse.

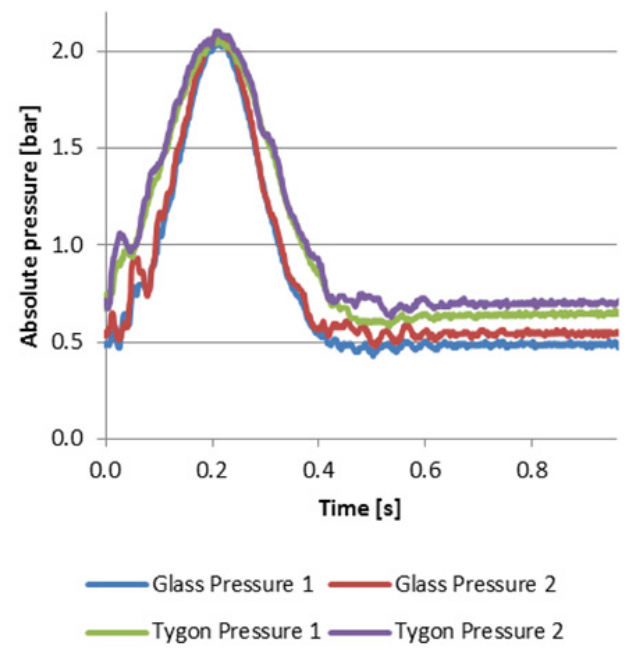

Fig. 3. The effect of flexible wall on the pressure response. Comparison of pressure course between glass and Tygon tubes.

There is also seen in Figure 3 that the maximal pressure peak was set equal for all measurement regimes to get comparable results of different materials. We used reducing valve set to 2.1 bar to ensure the same pressure peak. Differences in pressures course are mostly significant in the peak width and lower stabilised pressure value. The Tygon material absorbs part of pressure and expands its diameter. The pressure oscillations are stronger for Tygon, also caused by the flexible wall. The pressure oscillations at the lower peak of the measured pressure are caused by the uniform action of the liquid on the walls of the flexible tube.

The liquid flow can be divided into four regimes: The first regime corresponds to pressure increase. The liquid flow is accelerated. The maximal fluid velocities reach $440 \mathrm{~mm} / \mathrm{s}(\operatorname{Re} 8800)$

The second regime is the highest point of pressure increase, where physically occurs the maximum membrane inclination, and closure of the pumps valves. At this point, the fluid stagnates, resulting in slowing down of the flow rate to $70 \mathrm{~mm} / \mathrm{s}$ (Re 1400), and changing the characteristic velocity profile. This regime can be said as transient mode. The velocity profile is characterized with typical velocity decrease in the middle of cross section. This effect was found for both measurements - using rigid and flexible wall.

The other phase of liquid acceleration can be observed in the third regime. Here, the liquid is fed with the pump. This acceleration last during the pressure decrease. This regime is characteristic with turbulent profile shape, and maximal flow velocity reach $260 \mathrm{~mm} / \mathrm{s}(\operatorname{Re} 5200)$.

The fourth regime is observed since $6 / 5 \pi$ period, when the pressure drop and starts to be stabilized. Here we can observe the liquid flow slowdown that is close to laminar profile shape. The maximal velocities in this regime are $35 \mathrm{~mm} / \mathrm{s}(\operatorname{Re} 700)$.

The nature of the fluid flow at the corresponding times of synchronization is the same for rigid and flexible wall, so the maximum fluid flow velocities are similar, but the velocity profile varies especially close to the wall. An apparent difference is seen in comparing the rigid and flexible walls. Liquid interaction with the flexible wall occurs in the phase of slowing down the liquid flow, i.e. laminar regime and transient one.

Table 1. Maximal fluid velocity across the velocity profile and Reynolds Number of the liquid flow

\begin{tabular}{ccc}
\hline Time in Period & Max. Velocity $[\mathrm{mm} / \mathrm{s}]$ & Reynolds Number \\
$0 \mathrm{~ms}$ & 440 & 8800 \\
$100 \mathrm{~ms}-1 / 5 \pi$ & 380 & 7600 \\
$200 \mathrm{~ms}-2 / 5 \pi$ & 70 & 1400 \\
$300 \mathrm{~ms}-3 / 5 \pi$ & 220 & 4400 \\
$400 \mathrm{~ms}-4 / 5 \pi$ & 260 & 5200 \\
$500 \mathrm{~ms}-\pi$ & 140 & 2800 \\
$600 \mathrm{~ms}-6 / 5 \pi$ & 36 & 720 \\
$700 \mathrm{~ms}-7 / 5 \pi$ & 28 & 560 \\
$800 \mathrm{~ms}-8 / 5 \pi$ & 40 & 800 \\
$900 \mathrm{~ms}-9 / 5 \pi$ & 35 & 700 \\
\hline
\end{tabular}

The series of following charts (Fig. 4) represent velocity profiles of fluid flow. There is an oscillation of a pressure, which leads to the formation of vortex structures near the wall. These structures are highlighted by the elasticity of the wall. There is developed nonstationary backward vortex close to the wall, as the fluid flow velocity is very small. The prevailing dominant effect on the fluid flow comes from the released kinetic energy from the relaxing flexible walls, especially in the fourth regime of pressure course.

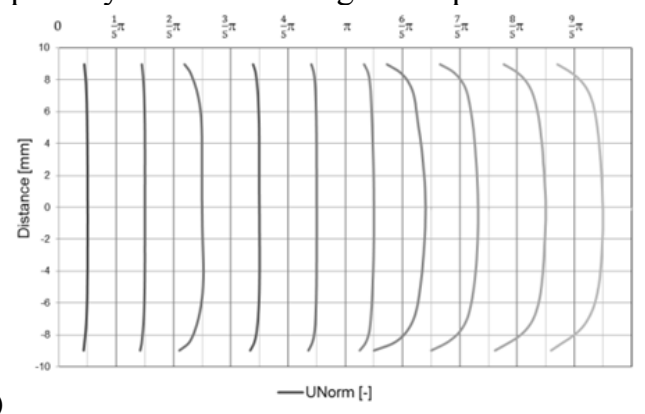




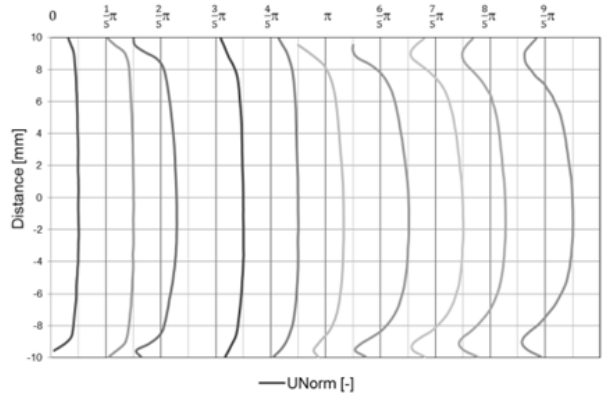

Fig. 4. Comparison of characteristic velocity profiles in the middle cross section using normalized velocity $U_{\text {NORM }}$ against maximal velocity of the fluid flow of a) glass tube, and b) Tygon flexible tube.

This effect is not significant for rigid walls. Here it is caused by very slow motion of fluid that generates tiny vortex structures that response to the fading shock wave from the membrane. There is generated backward flow, rapid reverse effect close to wall in this regime.

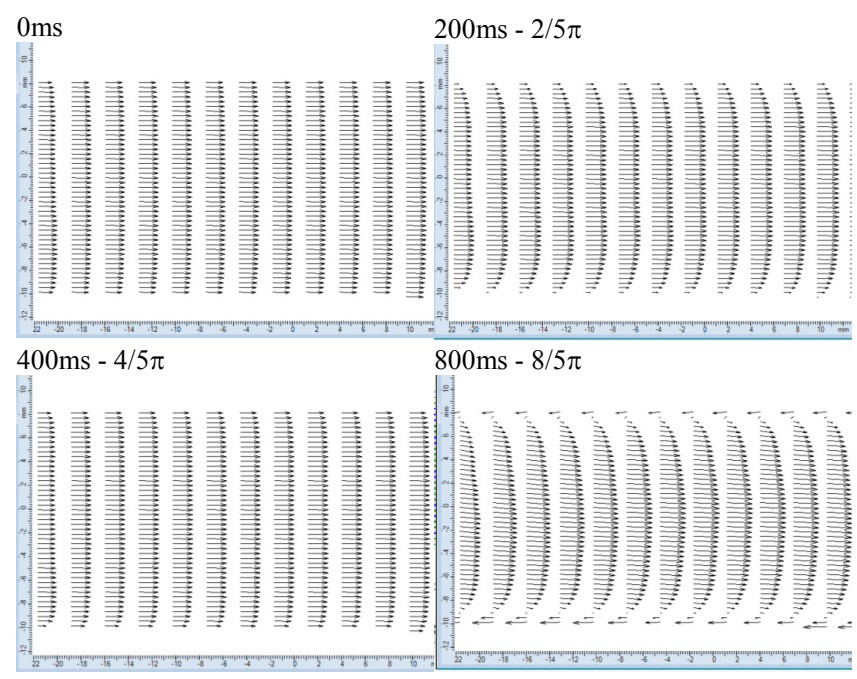

Fig. 5. Flow field of the liquid inside the rigid glass tubes.

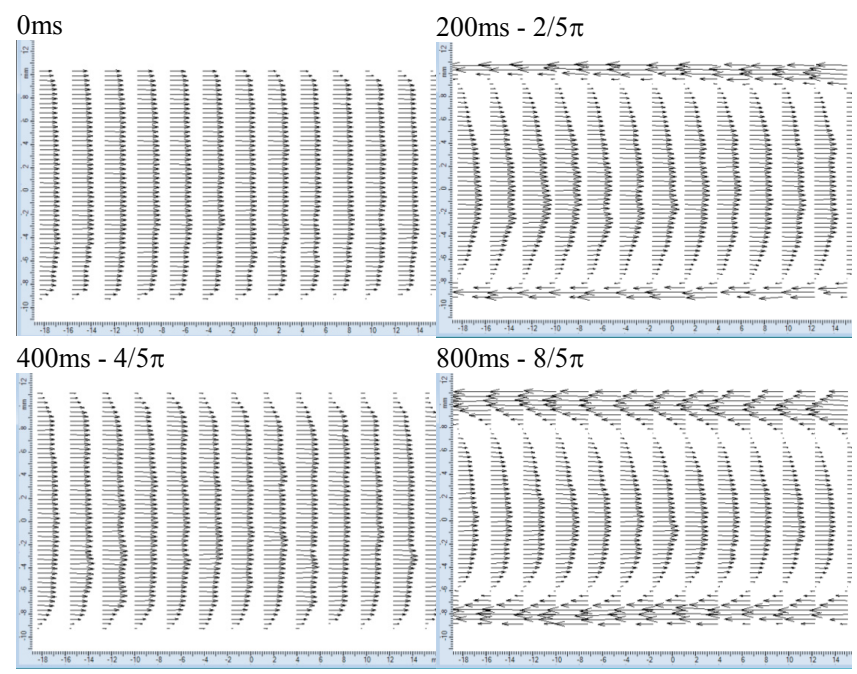

Fig. 6. Flow field of the liquid inside the flexible Tygon tube.

This phenomenon is physically explicable by the principle of closing the valves at the oscillation phase of the membrane. Vortex structures close to the wall can already be generated in the pump itself and spread through the pipeline due to the decaying pressure waves. The sudden change in the flow direction of the liquid will affect the region of slowest fluid flow, i.e. near the wall. Due to inertia, the main liquid stream does not slow down significantly, compared to the situation near the walls, where we expect the flow velocities to be lower, close to zero.

In general, the Newtonian fluid reaction is fast, which is confirmed by rapid transitions between the laminar, and turbulent fully developed velocity profile. The transition from the laminar to turbulent regime is followed by development of small disturbances. The laminar flow regime is unstable in time. The vector statistics that is seen in Fig. 5, and Fig. 6 are time averaged liquid flow characteristics. It can be said as a transfer from stable (turbulent) to unstable (laminar) mode. Here we can express the balance between the viscous liquid forces and elastic forces in the wall, when the flow close to wall becomes unstable followed by vortex structures.

Authors gratefully thank to the support of Grant Agency of the Czech Republic (GA ČR) 17-19444S Interaction of heterogeneous liquid with flexible wall, and LO1201 cofunding from the Ministry of Education, Youth and Sports as part of targeted support from the „National Programme for Sustainability I".

\section{Conclusions}

The measurement of velocity profiles has extreme importance for the verification of numerical simulation and validation of theories. The dominant role is in dynamic spatial range that should be focused on the region close to wall. The rough character of velocity profiles in each time step over the period was described and the effect of the flexible wall was expressed. The study of other materials, especially those used in healthcare will help them to evolve further, so their behaviour approaches the real organs.

\section{References}

1. D. A. McDonald, J. Physiol., 127 (1955).

2. C. G. Caro, J. Physiol., 157 (1691).

3. R. A. Womersley, J. Physiol., 127 (1955).

4. J. F. Hale, D. A. McDonald, J. R. Womersley, J. Physiol. 128 (1955).

5. F. Pochyly, et al., The wettability of the liquidsolid interface, 9th International Conference on FlowInduced Vibration (2008), pp. 47-52. 